Int. J. Contemp. Math. Sciences, Vol. 2, 2007, no. 22, 1077 - 1083

\title{
On Banach-Steinhause Theorems in Generalized 2-Normed Spaces
}

\author{
M. Açıkgöz and M. Menekse \\ University of Gaziantep, Faculty of Science and Arts \\ Department of Mathematics, 27310 Gaziantep, Turkey \\ acikgoz@gantep.edu.tr, menekse@gantep.edu.tr
}

Abstract. In this study, we will review Banach-Steinhause theorems of bounded linear operators from a normed space into a generalized 2-normed space. This study is a review on the three works of Zofia Lewandowska.

Mathematics Subject Classification: Primary 46A15, Secondary 41A65

Keywords: Generalized 2-normed space, Bounded 2-linear operators, BanachSteinhause theorems

\section{Introduction}

Definition 1. ([1] and [2]) Let $X$ and $Y$ be linear spaces, $D$ be non-empty subset of $X x Y$ such that for every $x \in X, y \in Y$ the sets

$$
D_{x}=\{y \in Y:(x, y) \in D\}, \quad D^{y}=\{x \in X:(x, y) \in D\}
$$

are linear subspaces of the spaces $Y$ and $X$, respectively. A function $\|.,$.$\| :$ $D \rightarrow[0, \infty)$ is called a generalized 2-norm on $D$ if it satisfies the following conditions:

(N1) $\|x, \alpha y\|=|\alpha| \cdot\|x, y\|=\|\alpha x, y\|$ for all $(x, y) \in D$ and every scalar $\alpha$,

(N2) $\|x, y+z\|=\|x, y\|+\|x, z\| \quad$ for all $(x, y),(x, z) \in D$,

(N3) $\|x+y, z\|=\|x, z\|+\|y, z\| \quad$ for all $(x, z),(y, z) \in D$,

Then $(D,\|.,\|$.$) is called a 2-normed set. In particular, if D=X \times X Y$, then $(X \mathrm{x} Y,\|.,\|$.$) is called a generalized 2-normed space. Moreover if X=Y$, then the generalized 2 -normed space is denoted by $(X,\|.,\|$.$) .$

Theorem 1. ([1] and [2]) Let $(X x Y,\|.,\|$.$) be a generalized 2-normed space.$

(a) A family $\beta$ of all sets defined by $\bigcap_{i=1}^{n}\left\{x \in X:\left\|x, y_{i}\right\|<\varepsilon\right\}$, where $n \in \mathbb{N}, y_{1}, \ldots, y_{n} \in Y$ and $\varepsilon>0$, forms a complete system of neighborhoods of zero for a locally convex topology in $Y$. 
(b) A family $\beta$ of all sets defined by $\bigcap_{i=1}^{n}\left\{y \in Y:\left\|x_{i}, y\right\|<\varepsilon\right\}$, where $n \in \mathbb{N}, x_{1}, \ldots, x_{n} \in X$ and $\varepsilon>0$, forms a complete system of neighborhoods of zero for a locally convex topology in $X$.

We will denote the above topologies by the symbols $\tau(X, Y)$ and $\tau(Y, X)$, respectively. In the case when $X=Y$, we will denote these topologies by $\tau_{1}(X)=\tau(X, Y)$ and $\tau_{2}(X)=\tau(Y, X)$.

Definition 2. (i) Let $(X x Y,\|.,\|$.$) be a generalized 2-normed space and \Sigma$ a directed set. A net $\left\{x_{\sigma}\right\}_{\sigma \in \Sigma}$ is convergent to $x_{0} \in X$ in $(X, \tau(X, Y))$ if and only if for all $y \in Y$ and each $\varepsilon>0$ there exists $\sigma_{0} \in \Sigma$ such that $\left\|x_{\sigma}-x_{0}, y\right\|<\varepsilon$ for all $\sigma \geq \sigma_{0}$. Also, a net $\left\{y_{\sigma}\right\}_{\sigma \in \Sigma}$ is convergent to $y_{0} \in Y$ in $(Y, \tau(Y, X))$ if and only if for all $x \in X$ and $\varepsilon>0$ there exists $\sigma_{0} \in \Sigma$ such that $\left\|x, y_{\sigma}-y_{0}\right\|<\varepsilon$ for all $\sigma \geq \sigma_{0}([2])$

(ii) Let $(X x Y,\|.,\|$.$) be generalized 2-normed space. A sequence \left\{x_{n}\right\}_{n \geq 1}$ in $X$ is called a Cauchy sequence if for every $y \in Y$ and $\varepsilon>0$ there exists a $n \in \mathbb{N}$ such that $\left\|x_{n}-x_{m}, y\right\|<\varepsilon$ for all $n, m \geq n_{0}$.

(iii) Let $(X x Y,\|.\|$,$) be generalized 2-normed space. The space (X, \tau(X, Y))$ is called sequentially complete if every Cauchy sequence in $X$ is convergent in this space.

(iv) Let $X$ be a real normed space and $\Gamma \subseteq Y x Y$ be a 2-normed set, where $Y$ denotes a real linear space. Define

$$
\begin{aligned}
F=\left\{(f, g) \in L(X, Y)^{2}: \forall x \in X \quad(f(x), g(x)) \in \Gamma\right. \\
\left.\& \exists M>0, \forall x \in X \quad\|f(x), g(x)\| \leq M .\|x\|^{2}\right\}
\end{aligned}
$$

Then, $(F,\|.,\|$.$) is a 2-normed set, where the function \|.\|:, F \longrightarrow[0, \infty)$ is defined by

$$
\|f, g\|=\inf \left\{M>0: \forall x \in X \quad\|f(x), g(x)\| \leq M .\|x\|^{2}\right\} .
$$

Note that the sets

$$
F^{g}=\left\{f^{\prime} \in L(X, Y):\left(f^{\prime}, g\right) \in F\right\} \& F_{f}=\left\{g^{\prime} \in L(X, Y):\left(f, g^{\prime}\right) \in F\right\}
$$

are linear subspaces of the space $L(X, Y)$, for every $(f, g) \in L(X, Y)^{2}$.

Similarly we can define

$$
\begin{aligned}
& \Theta=\left\{(f, g) \in L(X, Y)^{2}: \forall x \in X \quad(f(x), g(x)) \in \Gamma\right. \\
& \qquad \forall \exists M>0, \forall x \in X \quad\|f(x), g(y)\| \leq M .\|x\| .\|y\|\}
\end{aligned}
$$

Then, $(\Theta,\|.,\|$.$) is a 2-normed set, where the function \|.,\|:. \Theta \longrightarrow[0, \infty)$ is defined by

$$
\|f, g\|=\inf \{M>0: \forall x \in X \quad\|f(x), g(y)\| \leq M .\|x\| \cdot\|y\|\} .
$$


Similarly the sets

$$
\Theta^{g}=\left\{f^{\prime} \in L(X, Y):\left(f^{\prime}, g\right) \in \Theta\right\} \quad \mathscr{G} \Theta_{f}=\left\{g^{\prime} \in L(X, Y):\left(f, g^{\prime}\right) \in \Theta\right\}
$$

are linear subspaces of the space $L(X, Y)$, for every $(f, g) L(X, Y)^{2}$.

\section{Some Results}

Theorem 2. Let $(X,\|\|$.$) be a Banach space, (Y,\|.,\|$.$) be a generalized 2-$ normed space and $\left\{f_{n}\right\}_{n \geq 1}$ a sequence of elements from $\Theta^{g}$ for some $g \in$ $L(X, Y)$. Then the following conditions are equivalent:

(a) The sequence of 2-norms $\left\{\left\|f_{n}, g\right\|\right\}_{n>1}$ is bounded.

(b) $\exists M>0 \forall x, y \in X,\|x\| \leq 1,\|y\| \leq 1, \forall n \geq 1,\left\|f_{n}(x), g(y)\right\| \leq M$.

(c) The following conditions are true:

(i) $\forall x \in X, \exists M_{x}>0, \forall y \in X,\|y\| \leq 1, \forall n \geq 1,\left\|f_{n}(x), g(y)\right\| \leq M_{x}$.

(ii) $\forall y \in X, \exists M_{y}>0, \forall x \in X,\|x\| \leq 1, \forall n \geq 1,\left\|f_{n}(x), g(y)\right\| \leq M_{y}$.

Proof. At first, let us suppose that the sequence of 2-norms $\left\{\left\|f_{n}, g\right\|\right\}_{n \geq 1}$ is bounded. From this, it follows that there exists a positive number $M$ such that $\left\|f_{n}, g\right\| \leq M$ for each $n \in N$. Thus for $x, y \in X,\|x\| \leq 1,\|y\| \leq 1$ and $n \in N$, we obtain $\left\|f_{n}(x), g(y)\right\| \leq\left\|f_{n}, g\right\| \cdot\|x\| \cdot\|y\| \leq M$. Now, let the condition (b) be satisfied. We fix $x \in X \backslash\{0\}$. Then for each $y \in X,\|y\| \leq 1$ and $n \in N$ we obtain the inequalities:

$$
\left\|f_{n}(x), g(y)\right\|=\left\|f_{n}\left(\frac{x}{\|x\|} \cdot\|x\|\right), g(y)\right\|=\|x\| \cdot\left\|f_{n}\left(\frac{x}{\|x\|}, g(y)\right)\right\| \leq M .\|x\|
$$

If we choose $M_{x}=M .\|x\|$, then we have the condition (i). Moreover, for $x=0$ the condition (i) is satisfied for every positive number $M_{x}$. In similar way, taking $M_{y}=M .\|y\|$ for each $y \in X \backslash\{0\}$ and any positive number for $y=0$, we obtain ( $i i$ ). Conversely, let (i) and (ii) be satisfied. In $X x X$, let us define a norm by the formula,

$$
\|(x, y)\|_{*}=\|x\|+\|y\| \text { for each }(x, y) \in X x X .
$$

It is easy to verfy that $\left(X x X,\|\cdot\|_{*}\right)$ is a Banach space. Put

$$
A_{n m}=\left\{(x, y) \in X x X:\left\|f_{n}(x), g(y)\right\| \leq m\right\} \text { and } B_{m}=\bigcap_{n=1}^{\infty} A_{n m}
$$

for $m, n \in N$. We shall show that the sets $B_{m}$ are closed in $\left(X x X,\|.\|_{*}\right)$ for each $m \in N$. At first, we shall show that sets $A_{n m}$ are closed in this space. Let $m, n \in N$ and let $\left\{\left(x_{k}, y_{k}\right): k \in N\right\} \subset A_{n m}$ be a sequence converging to $\left(x^{\prime}, y^{\prime}\right) \in X x X$. Then

$$
\left\|f_{n}\left(x_{k}\right), g\left(y_{k}\right)\right\| \leq m \text { and }\left\|\left(x_{k}, y_{k}\right)-\left(x^{\prime}, y^{\prime}\right)\right\|_{*} \rightarrow 0, k \rightarrow \infty .
$$

The last condition is equivalent to the following: $\left\|x_{k}-x^{\prime}\right\| \rightarrow 0$ and $\left\|y_{k}-y^{\prime}\right\| \rightarrow$ 0 , which implies the convergence of the sequence $\left\{x_{k}: k \in N\right\},\left\{y_{k}: k \in N\right\}$. 
As a consequence these sequences are bounded. There exists $K>0$ such that the inequalities $\left\|x_{k}\right\| \leq K,\left\|y_{k}\right\| \leq K$ are true for each $k \in N$. Using these results we get

$$
\begin{aligned}
\left\|f_{n}\left(x^{\prime}\right), g\left(y^{\prime}\right)\right\| \leq & M+K .\left\|f_{n}, g\right\| \cdot\left\|x_{k}-x^{\prime}\right\|+K .\left\|f_{n}, g\right\| \cdot\left\|y_{k}-y^{\prime}\right\| \\
& +\left\|f_{n}, g\right\| \cdot\left\|x_{k}-x^{\prime}\right\| \cdot\left\|y_{k}-y^{\prime}\right\| .
\end{aligned}
$$

Letting $k \rightarrow \infty$, we obtain $\left\|f_{n}\left(x^{\prime}\right), g\left(y^{\prime}\right)\right\| \leq M$, which means that $\left(x^{\prime}, y^{\prime}\right) \in$ $A_{n m}$. Therefore the sets $A_{n m}$ are closed for each $n, m \in N$, and hence the sets $B_{m}$ are closed in $\left(X x X,\|\cdot\|_{*}\right)$.

Now we shall show that the equality $X x X=\bigcup_{m=1}^{\infty} B_{m}$ is true. Let $x, y \in X, x \neq 0$.Then $\left\|\frac{x}{\|x\|}\right\|=1$. By virtue $(i i)$ there exists $M_{y}>0$ such that $\left\|f_{n}\left(\frac{x}{\|x\|}\right), g_{y}\right\| \leq M_{y}$ for each $n \in N$. Thus $\left\|f_{n}(x), g(y)\right\| \leq M_{y} \cdot\|x\|$ for each $n \in N$.If $x=0$, then $\|x\| \leq 1$ and $\left\|f_{n}(x), g(y)\right\|=\|0, g(y)\|=0=M_{y} \cdot\|0\|$. as a consequence, for every $x, y \in X$ the sequence $\left\{\left\|f_{n}(x), g(y)\right\|: n \in N\right\}$ is bounded.From this it follows that for any point $(x, y) \in X x X$ there exists $m \in N$ such that $\left\|f_{n}(x), g(y)\right\| \leq M$ for every $m \in n$,i.e. $(x, y) \in \bigcup_{m=1}^{\infty} B_{m}$. Thus $X x X=\bigcup_{m=1}^{\infty} B_{m}$. By the well known Baire theorem there exists a set $B_{m_{0}}$ with non-empty interior. Therefore $B_{m_{0}}$ contains some closed ball with the center $\left(x_{0}, y_{0}\right)$ and radius $r$.Denote it by $K\left(\left(x_{0}, y_{0}\right), r\right)$. Thus for each $n \in N$ and $(x, y) \in K\left(\left(x_{0}, y_{0}\right), r\right)$, we have $\left\|f_{n}(x), g(y)\right\| \leq m_{0}$. Let us take $x, y \in X$ such that $\|x\| \leq \frac{r}{2}$ and $\|y\| \leq \frac{r}{2}$. Then

$\|(x, y)\|_{*}=\|x\|+\|y\| \leq r$ and $\|(x, y)\|_{*}=\left\|\left(x+x_{0}, y+y_{0}\right)-\left(x_{0}, y_{0}\right)\right\|_{*} \leq r$.

Therefore $\left\|f_{n}\left(x+x_{0}\right), g\left(y+y_{0}\right)\right\| \leq m_{0}$. In particular, $\left\|f_{n}\left(x_{0}\right), g\left(y_{0}\right)\right\| \leq$ $m_{0}$. Thus,

$$
\begin{aligned}
\left\|f_{n}(x), g(y)\right\| \leq & \left\|f_{n}\left(x+x_{0}\right), g\left(y+y_{0}\right)\right\|+\left\|f_{n}\left(x+x_{0}\right), g\left(y_{0}\right)\right\| \\
& +\left\|f_{n}\left(x_{0}\right), g\left(y+y_{0}\right)\right\|+\left\|f_{n}\left(x_{0}\right), g\left(y_{0}\right)\right\| \\
\leq & 2 m_{0}+\left\|f_{n}\left(x_{0}\right)+f_{n}\left(x_{0}\right), g\left(y_{0}\right)\right\|+\left\|f_{n}\left(x_{0}\right), g(y)+g\left(y_{0}\right)\right\| \\
\leq & 4 m_{0}+\left\|f_{n}(x), g\left(y_{0}\right)\right\|+\left\|f_{n}\left(x_{0}\right), g(y)\right\| .
\end{aligned}
$$

So we have shown that the inequalities $\|x\| \leq \frac{r}{2}$ and $\|y\| \leq \frac{r}{2}$ imply the condition

$$
\left\|f_{n}(x), g(y)\right\| \leq 4 m_{0}+\left\|f_{n}(x), g\left(y_{0}\right)\right\|+\left\|f_{n}\left(x_{0}\right), g(y)\right\| .
$$

Now, let $x, y \in X,\|x\| \leq 1$ and $\|y\| \leq 1$. Because $\left\|\frac{r}{2} x\right\| \leq \frac{r}{2}$ and $\left\|\frac{r}{2} y\right\| \leq \frac{r}{2}$, then

$$
\left\|f_{n}\left(\frac{r}{2} x\right), g\left(\frac{r}{2} y\right)\right\| \leq 4 m_{0}+\left\|f_{n}\left(\frac{r}{2} x\right), g\left(y_{0}\right)\right\|+\left\|f_{n}\left(x_{0}\right), g\left(\frac{r}{2} y\right)\right\| .
$$


As a sequence, we obtain

$\left\|f_{n}(x), g(y)\right\| \leq \frac{16 m_{0}}{r^{2}}+\frac{2}{r}\left(\left\|f_{n}(x), g\left(y_{0}\right)\right\|+\left\|f_{n}(x), g(y)\right\|\right) \quad$ for each $n \in N$.

Applying $(i)$ we have that there exists $M_{x_{0}}>0$ such that for every $y \in X$, $\|y\| \leq 1$ and $n \in N$ the inequality $\left\|f_{n}\left(x_{0}\right), g(y)\right\| \leq M_{x_{0}}$ is true. However the assumption (ii) implies there exists $M_{y_{0}}>0$ such that for every $x \in X$, $\|x\| \leq 1$ and $n \in N$ the inequality $\left\|f_{n}(x), g\left(y_{0}\right)\right\| \leq M_{y_{0}}$ is satisfied. So

$$
\left\|f_{n}(x), g(y)\right\| \leq \frac{16 m_{0}}{r^{2}}+\frac{2}{r}\left(M_{y_{0}}+M_{x_{0}}\right)
$$

for each $n \in N$ and $x, y \in X$ such that $\|x\| \leq 1,\|y\| \leq 1$.Therefore

$$
\begin{aligned}
\left\|f_{n}, g\right\| & =\sup \left\{\left\|f_{n}(x), g(y)\right\|: x, y \in X,\|x\| \leq 1,\|y\| \leq 1\right\} \\
& \leq \frac{16 m_{0}+2 r\left(M_{x_{0}}+M_{y_{0}}\right)}{r^{2}}
\end{aligned}
$$

for each $n \in N$. So the sequence $\left\{\left\|f_{n}, g\right\|: n \in N\right\}$ is bounded and the proof is complete.

Theorem 3. ([3]) Let $(X,\|\|$.$) be a Banach space, (Y,\|.,\|$.$) a generalized$ 2-normed space and $g$ a linear operator from $X$ to $Y$.If $\left\{f_{n}: n \in N\right\} \subset \theta^{g}$ is pointwise convergent to $f \in L(X, Y)$ and satisfies one of the conditions $(a),(b),(c)$ from Theorem 2.1 then $f \in \theta^{g}$.

Theorem 4. Let $A$ be a linearly dense set in a Banach space $(X,\|\|),.(Y,\|.,\|$. be a generalized 2-normed space such that $\left(Y, \tau_{1}(Y)\right)$ is a Hausdoff sequentially complete space. Let $g$ be a linear operator from $X$ into $Y$ and $\left\{f_{n}\right\}_{n \geq 1} \subseteq \Theta^{g}$. Then the following conditions are equivalent:

(a) The sequence $\left\{f_{n}\right\}_{n>1}$ is pointwise convergent to $f \in L(X, Y)$ and the conditions (i), (ii) from Theorem 2 are satisfied.

(b) The sequence $\left\{f_{n}\right\}_{n \geq 1}$ is pointwise convergent to $f \in \Theta^{g}$ on the set $A$ and the sequence of 2 -norms $\left\{\left\|f_{n}, g\right\|\right\}_{n \geq 1}$ is bounded.

Proof. If the sequence $\left\{f_{n}(x): n \in N\right\}$ is convergent to $f(x) \in Y$ for each $x \in X$, then it convergent also for $x \in A \subset X$. Moreover this follows from theorem 2.1 and theorem 3-the sequence $\left\{\left\|f_{n}, g\right\|: n \in N\right\}$ is bounded and $f \in \theta^{g}$. Now, we will suppose that the sequence $\left\{f_{n}: n \in N\right\}$ is pointwise convergent to $f \in \theta^{g}$ on the set $A$ and the conditions $(i),(i i)$ hold. Let $X_{0}$ be the vector subspace of the Banach space $X$ generated by $A$. So $X_{0}$ is normed space. Let $x, y \in X_{0}$. Then $x=a_{1} x_{1}+\ldots+a_{k} x_{k}, y=b_{1} y_{1}+\ldots+b_{t} y_{t}$, where $a_{i}, b_{j} \in R, x_{i}, y_{j} \in A, i=1,2,3, \ldots, k ; j=1,2,3, \ldots, t$ and $k, t \in N$. Thus, it follows from assumptions on $f_{n}, f, g$ that $\left\|f_{n}(x)-f(x), g(y)\right\|=$ $\left\|a_{1}\left(f_{n}\left(x_{1}\right)-f\left(x_{1}\right)\right)+\ldots+a_{k}\left(f_{n}\left(x_{k}\right)-f\left(x_{k}\right)\right), b_{1} g\left(y_{1}\right)+b_{2} g\left(y_{2}\right)+\ldots+b_{t} g\left(y_{t}\right)\right\|$. 
Using properties of 2-norms, we get

$$
\left\|f_{n}(x)-f(x), g(y)\right\| \leq \sum_{i=1}^{k} \sum_{j=1}^{t}\left|a_{i} b_{j}\right| .\left\|f_{n}\left(x_{i}\right)-f\left(x_{i}\right), g\left(y_{j}\right)\right\| .
$$

Because $\lim _{n \rightarrow \infty}\left\|f_{n}\left(x_{i}\right)-f\left(x_{i}\right), g\left(y_{j}\right)\right\|=0$ for ach $x_{i}, y_{j} \in A$,

then $\lim _{n \rightarrow \infty}\left\|f_{n}(x)-f(x), g(y)\right\|=0$.i.e. the sequence $\left\{f_{n}: n \in N\right\}$ is convergent to $f$ on $X_{0}$. Let $\left\|f_{n}, g\right\| \leq M$ for every $n \in N$. Let us take a number $\varepsilon>0, x \in X$ and $y \in X$ such that $y \neq 0$. Since $X_{0}$ is a dense set in $X$, we can choose $x_{0} \in X_{0}, x_{0} \neq 0$ such that $\left\|x-x_{0}\right\|<\frac{\varepsilon}{6 . M .\|y\|}$. Moreover, there exists $y_{0} \in X_{0}$ with the property $\left\|y-y_{0}\right\|<\frac{\varepsilon}{6 . M .\left\|x_{0}\right\|}$. The sequence $\left\{f_{n}\left(x_{0}\right): n \in N\right\}$ is convergent in $\left(Y, \tau_{1}(Y)\right)$, so it is a Cauchy sequence in this space. Therefore, there exists a number $n_{0}$ such that $\left\|f_{n}\left(x_{0}\right)-f_{m}\left(x_{0}\right), g\left(y_{0}\right)\right\|<\frac{\varepsilon}{3}$ for each $n, m \geq n_{0}$. As a consequence, we obtain,

$$
\begin{aligned}
\left\|f_{n}(x)-f_{m}(x), g(y)\right\| \leq & \left\|f_{n}(x)-f_{n}\left(x_{0}\right), g(y)\right\|+\left\|f_{n}\left(x_{0}\right)-f_{m}\left(x_{0}\right), g\left(y_{0}\right)\right\| \\
& +\left\|f_{m}\left(x_{0}\right)-f_{m}(x), g(y)\right\| \\
\leq & \left\|f_{n}, g\right\| \cdot\left\|x-x_{0}\right\| \cdot\|y\|+\left\|f_{n}\left(x_{0}\right)-f_{m}\left(x_{0}\right), g\left(y-y_{0}\right)+g\left(y_{0}\right)\right\| \\
& +\left\|f_{m}, g\right\| \cdot\left\|x-x_{0}\right\| \cdot\|y\| \\
\leq & 2 M \cdot\left\|x-x_{0}\right\| \cdot\|y\|+\left\|f_{n}\left(x_{0}\right)-f_{m}\left(x_{0}\right), g\left(y-y_{0}\right)\right\| \\
& +\left\|f_{n}\left(x_{0}\right)-f_{m}\left(x_{0}\right), g\left(y_{0}\right)\right\| \\
< & 2 M \cdot\left\|x-x_{0}\right\| \cdot\|y\|+\left\|f_{n}\left(x_{0}\right), g\left(y-y_{0}\right)\right\| \\
& +\left\|f_{m}\left(x_{0}\right), g\left(y-y_{0}\right)\right\|+\frac{\varepsilon}{3} \\
< & 2 M \cdot \frac{\varepsilon}{6 M\|y\|} \cdot\|y\|+\left\|f_{n}, g\right\| \cdot\left\|x_{0}\right\| \cdot\left\|y-y_{0}\right\| \\
& +\left\|f_{m}, g\right\| \cdot\left\|x_{0}\right\| \cdot\left\|y-y_{0}\right\| \\
< & \frac{2}{3} \varepsilon+2 M\left\|x_{0}\right\| \cdot \frac{\varepsilon}{6 M\left\|x_{0}\right\|}=\varepsilon \quad \text { for } n, m \geq n_{0} .
\end{aligned}
$$

If $y=0$, then the inequality $\left\|f_{n}(x)-f_{m}(x), g(y)\right\|=0<\varepsilon$ is also true. Hence we have shown that $\left\{f_{n}(x): n \in N\right\}$ is a Cauchy sequence in $\left(Y, \tau_{1}(Y)\right)$ for every $x \in X$. Because $\left(Y, \tau_{1}(Y)\right)$ is a sequentially complete space, then the sequence $\left\{f_{n}: n \in N\right\}$ is pointwise convergent. Let us denote $h(x)=$ $\lim _{n \rightarrow \infty} f_{n}(x)$ for every $x \in X$. The fact that $\left(Y, \tau_{1}(Y)\right)$ is a Hausdorff space implies $h(x)=f(x)$ for $x \in A$, i.e. $(h-f)(x)$ for $x \in A$. The operator $h-f$ is linear, thus $(h-f)(x)=0$ for every $x \in X_{0}$. Using theorem 3 , we see that $h \in \theta^{g}$. Because $\theta^{g}$ is a linear subspace, then $h-f \in \theta^{g}$. Thus there exists a positive number $K$ such that

$$
\|(h-f)(x), g(y)\| \leq K .\|x\| .\|y\| \text { for every } x, y \in X
$$


Let $\varepsilon>0, x, y \in X, y \neq 0$. Since the set $X_{0}$ is dense in $X$, we can choose $x_{0} \in X_{0}$ such that $\left\|x-x_{0}\right\|<\frac{\varepsilon}{K .\|y\|}$. Then

$$
\begin{aligned}
0 & \leq\|(h-f)(x), g(y)\|=\left\|(h-f)\left(x-x_{0}\right)+(h-f)\left(x_{0}\right), g(y)\right\| \\
& =\left\|(h-f)\left(x-x_{0}\right), g(y)\right\| \\
& \leq K .\left\|x-x_{0}\right\| \cdot\|y\|<\varepsilon .
\end{aligned}
$$

This gives $\|(h-f)(x), g(y)\|=0$ for each $x \in X, y \in X \backslash\{0\}$. Thus $h(x)=$ $f(x)$ for every $x \in X$. As a consequence we have shown that the sequence $\left\{f_{n}: n \in N\right\}$ is pointwise convergent to $f$, which finishes the proof.

Theorem 5. Let $(X,\|\|$.$) be a Banach space, (Y,\|.,\|$.$) be a generalized 2-$ normed space and $\left\{\left(f_{n}, g_{n}\right)\right\}_{n \geq 1}$ a sequence of elements from $\Theta$. Then the following conditions are equivalent:

(a) The sequence of 2-norms $\left\{\left\|f_{n}, g_{n}\right\|\right\}_{n \geq 1}$ is bounded.

(b) $\exists M>0 \forall x, y \in X,\|x\| \leq 1,\|y\| \leq 1, \forall n \geq 1,\left\|f_{n}(x), g_{n}(y)\right\| \leq M$.

(c) The following conditions are satisfied:

(i) $\forall x \in X, \exists M_{x}>0, \forall y \in X,\|y\| \leq 1, \forall n \geq 1,\left\|f_{n}(x), g_{n}(y)\right\| \leq M_{x}$.

(ii) $\forall y \in X, \exists M_{y}>0, \forall x \in X,\|x\| \leq 1, \forall n \geq 1,\left\|f_{n}(x), g_{n}(y)\right\| \leq M_{y}$.

\section{REFERENCES}

[1] Z. Lewandowska, Linear operators on generalized 2-normed spaces, Bull. Math. Soc. Sci. Math. Roumanie (N.S.) 42(90), no.4 (1999), 353-368.

[2] Z. Lewandowska, Z., On 2-normed sets, Glas.Math.Ser.III, 38(58), no.1, (2003) 99-110.

[3] Z. Lewandowska, Z., (2003), Banach Steinhaus theorems for bounded linear operators with values in a generalized 2-normed space, Glas. Mat. Ser.III, 38(58), no.2, 329-340.

Received: March 23, 2007 